\title{
PHYSICAL APPROACH TO TRANSDERMAL DRUG DELIVERY: A REVIEW
}

\author{
Jawale N.R. ", Bhangale C.D., Chaudhari M.A., Dr Deshmukh T.A.
}

SES Arunamai College of Pharmacy, Jalgaon, M.H., India

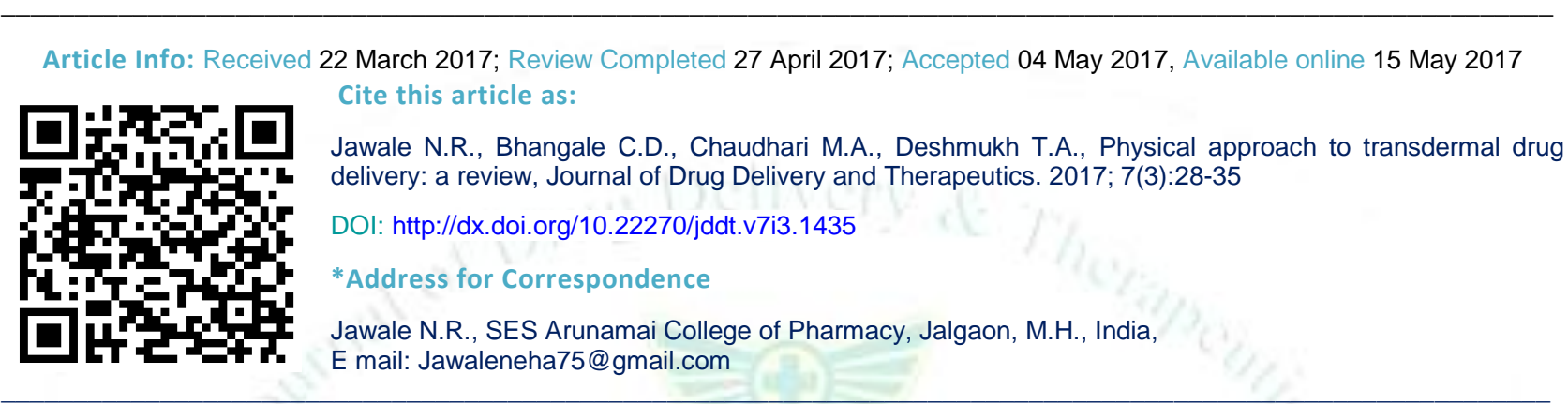

\begin{abstract}
Transdermal drug delivery system (TDDS) is the topically administered medication, which when applied to the skin, delivers the drug through the skin for systemic effect at a predetermined and controlled rate. For the drugs, having short biological half life, transdermal drug delivery system is convenient. It overcome the problems like the hepatic first pass effect, improves the compliance of patients, decreases the administration frequency, and reduce the gastrointestinal side effects, peak and valley levels in the blood are avoided. Due to have an advantage of being non-invasive, this delivery has to fulfill some parameters such as high potency, better permeability through skin and non-irritation for better compliance. Skin serves as a site of drug application for local effect as well as systemic effect. There are wide varieties of drugs for which topical or transdermal is a viable option, skin penetration enhancement techniques have been developed for such drugs. There are a number of physical methods to increase drug delivery via the skin, many of which require the use of devices and some of which hold the promise to significantly expand the spectrum of drugs that can be administered transdermally to include water-soluble molecules and macromolecules. Iontophoresis and electroporation represent electrically assisted, physical approaches to enhance delivery of drugs/macromolecules across the stratum corneum. This review highlights the detailed role of physical penetration and describes their mechanism of action.
\end{abstract}

Keywords: transdermal drug delivery system, stratum corneum, skin penetration enhancer, physical enhancer, iontophoresis.

\section{INTRODUCTION}

All conventional oral dosage form is required to be administered in multiple doses at a particular amount for an effective therapy. But it has limitations in case of patient showing symptoms of vomiting and diarrhoea cannot consume drugs by oral route. It is not suitable in cases of unconscious and in-cooperative patients. Some other drawbacks are inconvenient administration, chances of overdose if administered prior to time interval, lack of patient compliance, skip of dose by the patient, fluctuation of drug plasma level. Particular amount of drug is required for effective therapeutic uses. To avoid such complications transdermal drug delivery system (TDDS) are designed. Transdermal drug delivery system aid to development in delivery of proteins and neucleotides as the trend for discovering and designing biopharmaceuticals continues improvements in transdermal delivery will remain incremental until there is wider acceptance of this route of administration within the pharmaceutical industry. The main aim of transdermal drug delivery system is to provide prolong action of the drug with low toxicity and better patient compliance and thus reduces the side effects caused by oral route.

Transdermal drug delivery system is the system in which the delivery of the active ingredients of a drug occurs penetrate through the skin. This system is designed to improve the therapeutic efficacy and safety of the drugs 
because drug delivered through the skin at a predetermined and controlled rate.

In recent years, the use of a number of biophysical techniques has aided in our understanding of the nature of the stratum corneum barrier and the way in which chemicals interact with and influence this structure. A better understanding of the interaction of enhancers with the stratum corneum and the development of structure activity relationships for enhancers will aid in the design of enhancers with optimal characteristics and minimal toxicity.

In order to increase the range of drugs available for transdermal delivery the use of physical enhancement techniques have been developed in an attempt to compromise skin barrier function in a reversible manner without concomitant skin irritation. Several alternative physical methods have emerged to transiently break the stratum corneum barrier ${ }^{1,2,3}$.

The various advantages and disadvantages of transdermal drug delivery systems are given below:

\section{Advantages}

- Drug can be delivered over a long period of time.

- Avoidance of 'first-pass' metabolism of drugs.

- Adverse effects or therapeutic failures frequently associated with intermittent dosing can also be avoided.

- Reduction of fluctuations in plasma levels of drugs, Utilization of drug candidates with short half-life and low therapeutic index.

- Maintains stable or constant and controlled blood levels for longer period of time.

- Increased convenience to administer drugs which would otherwise require frequent dosing.

- Ability to discontinue administration by removal of the system.

- Self administration can be done.

Disadvantages of TDDS

- Drugs having affinity for both lipophilic and hydrophilicphases are used.

- Drugs in high concentration may cause skin irritation.

- Drugs with very low or high partition coefficient fail to reach systemic circulation.

- Difficult to administer large dose i.e. more than $10 \mathrm{mg} /$ day.

- $\quad$ Ionic drugs create problem.

\section{THE SKIN SITE FOR TRANSDERMAL ADMINISTRATION}

The skin, as the largest organ of the body, account for more than $10 \%$ of body mass and receives about one third of the blood circulating through the body, serves as a protective layer of the underlying tissues such as muscles, ligaments and internal organs, shielding it from exogenous molecules as well as from mechanical and radiation-induced injuries. The skin also plays a role in immunology and metabolism, regulates body temperature, serves as an excretory organ through sebaceous and sweat glands and contains sensory nerve endings for the perception of touch, temperature, pain and pressure. The skin varies in color, thickness and presence of nails, hairs and glands between the different regions of the body, although all types of skin have the same basic structure ${ }^{4,5}$

The skin can be considered to have four distinct layers of tissue ${ }^{6,7}$.

1. Non-viable epidermis (stratum corneum)

2. Viable epidermis

3. Viable dermis

4. Subcutaneous connective tissue (hypodermis)

Non-viable epidermis (stratum corneum)

Stratum corneum is the outer most layer of skin, which is the actual physical barrier to most substance that comes in contact with the skin. The stratum corneum is 10 to 20 cell layer thick over most of the body. Each cell is a flat, plate-like structure 34-44 $\mu \mathrm{m}$ long, 25-36 $\mu \mathrm{m}$ wide, 0.5 to $0.20 \mu \mathrm{m}$ thick - with a surface area of 750 to $1200 \mu \mathrm{m}^{2}$ stocked up to each other in brick like fashion. Stratum corneum consists of lipid (5-15\%) including phospholipids, glycolsphingolipid, cholesterol sulfate and neutral lipid, protein (75-85\%) which is mainly keratin.

\section{Viable epidermis}

This layer of the skin resides between the stratum corneum and the dermis and has a thickness ranging from 50- $100 \mu \mathrm{m}$. The structure of the cells in the viable epidermis is physiochemically similar to other living tissues. Cells are held together by tonofibrils. The density of this region is not much different than water. The water content is about $90 \%$.

\section{Dermis}

Just beneath the viable epidermis is the dermis. It is a structural fibrin and very few cells are like it can be found histological in normal tissue. Dermis thickness range from 2000 to $3000 \mu \mathrm{m}$ and consists of a matrix of loose connective tissue composed of fibrous protein embedded in an amorphous ground substance.

\section{Subcutaneous connective tissue}

The subcutaneous tissue or hypodermis is not actually considered a true part of the structured connective tissue is composed of loose textured, white, fibrous connective tissue containing blood and lymph vessels, secretory pores of the sweat gland and coetaneous nerves. Most investigators consider drug permeating through the skin enter the circulatory system before reaching the hypodermis, although the fatty tissue could serve as a depot of the drug. 


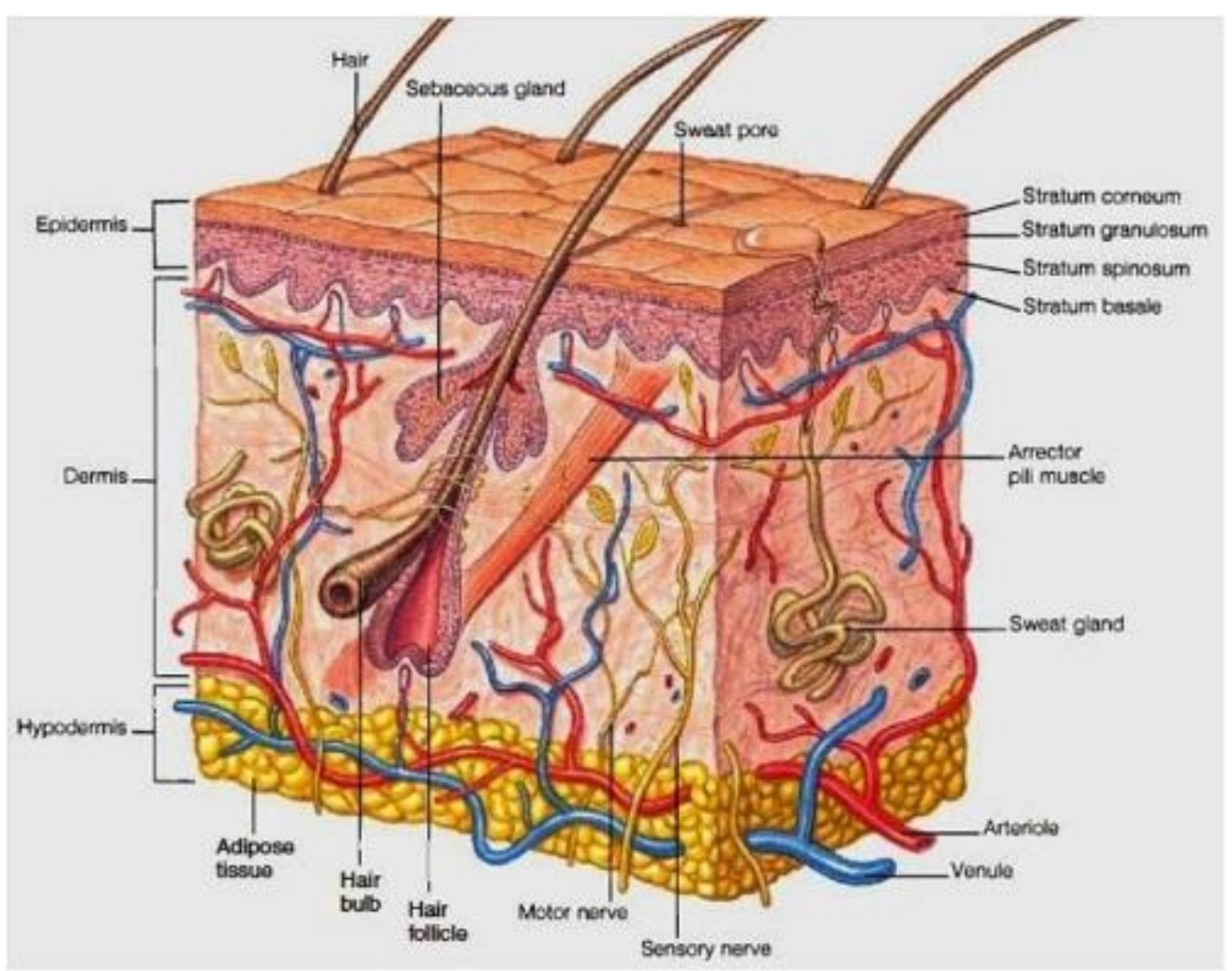

Figure 1: structure of skin ${ }^{12}$

\section{TRANSDERMAL DRUG DELIVERY SYSTEMS IN DRUG TARGETTING}

The transdermal drug delivery systems are used to target the drugs for purposes, described under ${ }^{1}$ :

\section{Surface of skin:}

Surface of skin is targeted for locally acting substances like disinfectants, cosmetics, insect repellent etc. in which drug acts only on the surface of the skin and no penetration of drug or chemicals in the skin.

\section{Skin layers itself:}

The delivery of drug substances within the skin layers is also known as topical delivery and skin layers are targeted when disease or infection is present in skin itself e.g. microbial infection, inflammation of skin and neoplasias etc.

\section{Systemic Circulation:}

It is considered as an alternative to oral and other conventional delivery routes for systemic delivery of drugs. The drug has to be permeated through the various skin layers.

\section{PATHWAYS OF DRUG ABSORPTION THROUGH THE SKIN}

The drug can be absorbed by various pathways through the skin depending on the physicochemical properties of the $\operatorname{drug}^{9,10,11}$. These are following;

\section{Transfollicular route}

Transfollicular route is the shortest pathway that drug has to follow to reach the systemic circulation that provides a large area for diffusion of drugs. Skin has various sweat glands, oil glands, hair follicles and pores opening to the outer surface of the skin via their ducts. These ducts offer a continuous channel across the stratum corneum for drug transport but various factors like secretion from glands, content and amount of secretion etc., affect the transport of drugs through this route. ${ }^{1}$

\section{Transcellular route}

Drugs entering the skin via the transcellular route pass through corneocytes. Corneocytes, containing highly hydrated keratin, provide an aqueous environment for which hydrophilic drugs can pass. The cells are surrounded by a lipid envelope which connects the cells to the interstitial lipids. Separating keratinised skin cells are multiple lipid bilayers; there are estimated to be up to 20 such lamellae between each corneocyte. Therefore, the diffusion pathway for a drug via the transcellular route requires a number of partitioning and diffusion steps. After partitioning into and diffusing through the relatively aqueous corneocytes, the permeant must partition into the surrounding lipid envelope, and subsequently partition in and out of the multiple lipid bilayers separating corneocytes .

Intercellular route

As name indicates in intercellular pathway the drug diffuses through the continuous lipid matrix present between the cells. The barrier property of this route is due tortuous structure formed by corneocytes and the drug has to pass through the alternating lipid and aqueous domain by partitioning into the lipid bilayer and diffusing to the inner side. It has been found that water has to travel 50 times more by this route so; it is suitable mainly for uncharged lipophilic drugs. 


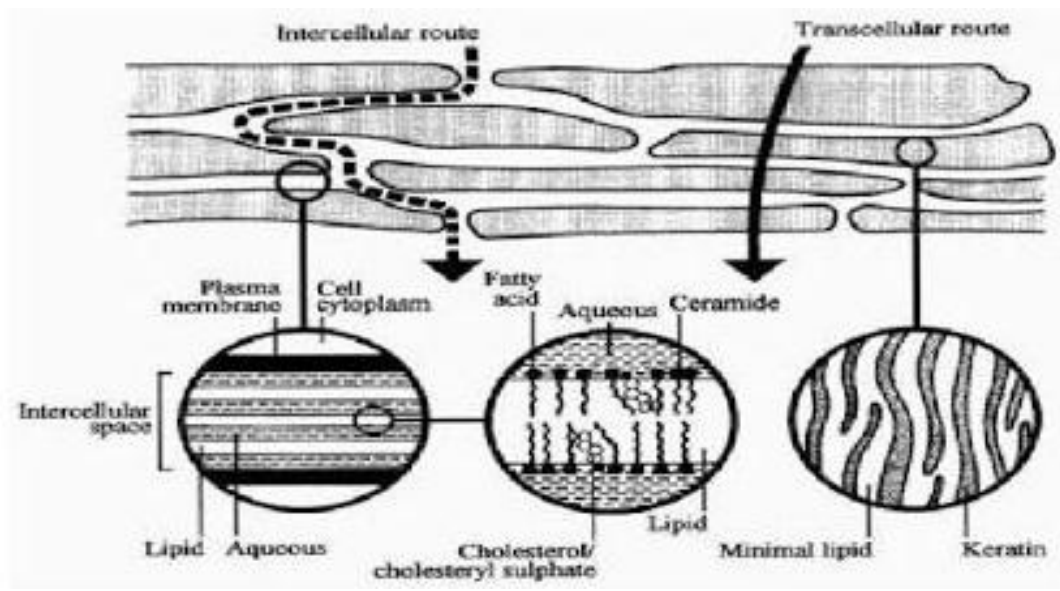

Figure 2: Pathways of permeation through skin

\section{PENETRATION ENHANCER}

Penetration enhancers are the substances used to increase permeation of skin mucosa. Penetration enhancer increases the absorption of penetrant through the skin which is also known as absorption promoter or absorption enhancers. Penetration enhancers used to increase the permeability of drug through $\operatorname{skin}^{8}$.

The penetration enhancers may show their effect any one or combination of the following mechanisms.

- By disrupting the structure of stratum corneum lipids.

- By interacting with intercellular proteins.

- By improving drug partitioning, co-enhancer or solvent into the stratum corneum.

- Dissolution of drug in its vehicle.

- Diffusion of drug from vehicle to surface of skin.

Ideal characteristics of enhancers

- An ideal penetration enhancer should have following properties.

- The effect of enhancer on the skin should be reversible, and it should not damage the viable cells.

- It should be pharmacologically inert, non-toxic, non-allergenic and non-irritating.

- It should be compatible with the drug and other additives.

- It should show their effect rapidly; with predictable and reproducible duration of effect.

- It should have unidirectional enhancing effect; allowing the drug molecule to pass through the skin while preventing the loss of endogenous materials like body fluids, electrolytes etc.

- It should be economical.

- No single penetration enhancer possesses all these properties; however some penetration enhancers may possesses some of the above attributes

\section{FACTORS AFFECTING TRANSDERMAL PERMEABILITY}

Various factors are involved in controlling and rendering permeability of drugs through the skin. These factors may be drug related or skin physiology related. Not only a single reason is responsible in affecting the permeation of drugs but a number of factors are involved which are mutually dependent on each other and are classified below:

1) Physiochemical properties of drug.

2) Formulation characteristics.

3) Skin physiology and pathology.

1) Physicochemical properties of drug

The various physicochemical properties of drug which can alter the absorption and diffusion of drug through the skin are:

a. Size of drug molecules and molecular weight

Size of drug molecules varies inversely to the penetration through the skin. Drug molecules larger than 500 dalton creates problem in percutaneous transport. Greater the molecular weight smaller is the absorption. So size of drug molecules should not be so high that it creates problem in absorption ${ }^{15}$.

\section{b. Partition coefficient and solubility}

Drugs are either lipophilc or hydrophilic in nature. The partition co-efficient determine the solubility or diffusion of drug in lipids and aqueous systems. Drugs possessing both lipid and water solubility are suitable for percutaneous absorption as skin is made of lipid bilayer so drug should have some lipid solubility for absorption but at same time it should have some hydrophilicity to diffuse inside the skin in aqueous environment. So a drug candidate should have optimum partition coefficient. The partition coefficient of a drug can be altered by changing the solvent system or by chemical modification in the structure of the drug candidate without affecting its pharmacological activity of the $\operatorname{drug}^{15}$.

\section{c. Drug concentration}

The absorption of drugs through the skin is governed by passive diffusion. Drug moves according to concentration gradient i.e. from high concentration to low concentration. So the concentration of drug in the formulation applied over the skin determines the 
diffusion rate across the skin. Higher the concentration more will be the permeation. ${ }^{15}$

\section{d. $\mathrm{pH}$ conditions}

Most of the drugs are either acidic or basic in nature. So the $\mathrm{pH}$ of the drug molecule determines its ionization at skin surface. Unionized drugs or species have better absorption than ions or ionic species, so $\mathrm{pH}$ plays an important role in determining extent of penetration of $\operatorname{drug}^{16}$.

\section{2) Formulation characteristics}

The various formulation characteristics can also alter the permeation of drug molecule through the skin. These are as follows:

\section{a. Release rate of the drug}

The release of drug from the formulation is influenced by the affinity of the carrier for the drug in formulation and physiochemical properties of drug like solubility of drug in solvent and interfacial partitioning of drug from formulation to skin determines the release rate of the $\operatorname{drug}^{16}$.

\section{b. Ingredients of formulation}

Various excipients and polymers present in the formulation can affect either release of drug or permeation of drug through the skin by altering the physicochemical properties of drug or skin physiology ${ }^{18}$.

\section{c. Presence of permeation enhancers}

Permeation enhancers of different categories are used to increase the permeation of drug through the skin. These alter the integrity of the skin (physicochemical and physiological modification) temporarily and open the skin pores for absorption. Permeation enhancer may be chemical substance which act chemically or physical permeation enhancer which physically interact with the skin integrity ${ }^{18}$.

3) Physiological and pathological condition of the skin

The physiological and pathological conditions of the skin alter and affect the permeation of drug candidate through the skin by changing the properties of the skin 17

\section{a. Hydration of skin}

Hydration of the skin causes the swelling of stratum corneum of the skin and provides some fluidity to the skin. Hydration also increases the permeant solubility and partitioning from vehicle to the membrane. So the permeation of drug molecules occurs easily through the hydrated $\operatorname{skin}^{17}$.

\section{b. Skin temperature}

On increasing the temperature of the skin the percutaneous absorption of the drug increases due to fluidization of lipids and vasodilation of the blood vessels which are in contact with the skin so increase in blood flow to the skin increases the absorption through the skin.
It is assumed that skin of young and elderly are more permeable than middle aged persons. In premature infants stratum corneum is absent and children are more susceptible to toxic effects of drugs through the skin.

\section{d. Blood flow}

Changes in peripheral circulation do not affect transdermal absorption butan increase in blood flow increase the concentration gradient across the skin and reduces the total time of residence of the drug molecules in the dermis by continuously removing it.

\section{e. Pathology of the skin}

Disease of the skin and any injury to the skin causes the rupturing of the lipid layers of the stratum corneum which alters the skin penetration of drugs. Pathogens cause the disruption of skin layers by digesting them and can create pores in the skin so the integrity of the skin changes in both pathological conditions and in injury ${ }^{17}$.

\section{f. Regional Site of skin}

The skin differs in anatomical features such as thickness of stratum corneum, number of hair follicles and number of sweat glands per unit surface area. This difference may exist from site to site, person to person and species to species. So in all cases percutaneous absorption differs from one another ${ }^{18}$.

\section{g. Skin flora and enzymes}

Various metabolizing enzymes and metabolizing microbes are present in the skin which metabolizes the drugs passing through the skin. Only a few drug candidates are there which reaches in active form in the circulation otherwise drugs are metabolized to various extents in the skin. E.g., $95 \%$ of the testosterone absorbed gets metabolized in the $\operatorname{skin}^{18}$.

\section{PHYSICAL APPROACH TO TRANSDERMAL DRUG DELIVERY SYSTEM}

Physical transdermal delivery enhancing methods increase the transdermal delivery by using an external energy as a driving force. Active methods make possible the permeation of larger molecules which can't be permeated otherwise. Various physical methods used for enhancing transdermal delivery are discussed here below;

\section{Iontophoresis}

It was developed to facilitate the delivery of ionized solute, with inherently low partition coefficients due to their charged states, across tissue membrane The principle of iontophoresis technique is based on that the like charges repels each other and opposite charge attract. Thus during iontophoresis, if delivery of positively charged drug, the charged drug is dissolved in the electrolyte surroundings the electrode of similar polarity, i.e. anode. An application of electromotive force the drug is repelled and moves across the stratum corneum towards the cathode, which is placed elsewhere on the body ${ }^{12,19,20,25}$ The drug permeation is caused by any one or combination of following mechanisms. 
- Electro-repulsion (for similar charged solutes)

- Electro-osmosis (for uncharged solutes)

- Electro-perturbation (for both charged and uncharged solutes)

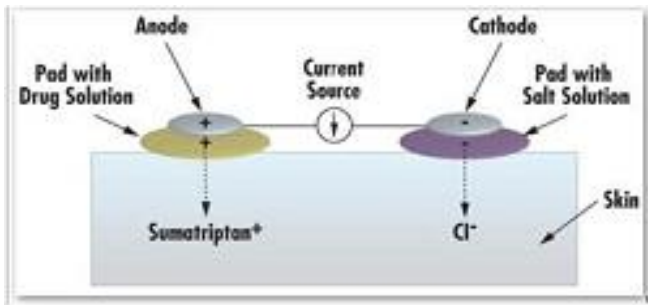

Figure 3: Diagrammatic representation of Iontophoresis with modification. ${ }^{14}$

\section{Sonophoresis}

It is the application of ultrasound to enhance the percutaneous drug delivery. In this method the drug is mixed with a coupling agent in the form of gel, cream or ointment. The coupling agent acts as a medium for the transfer of phonophoretic energy from the device to the skin. Sonophoresis causes disruption of stratum corneum by cavitation, microscreening and heat sensitization ${ }^{23,24}$. This involves rupturing the lipids present in stratum cornea, which allows the medicament to permeate via biological barrier ${ }^{26}$. It uses low frequency ultrasound $(55 \mathrm{kHz})$ for an average duration of 15 seconds to enhance skin permeability.

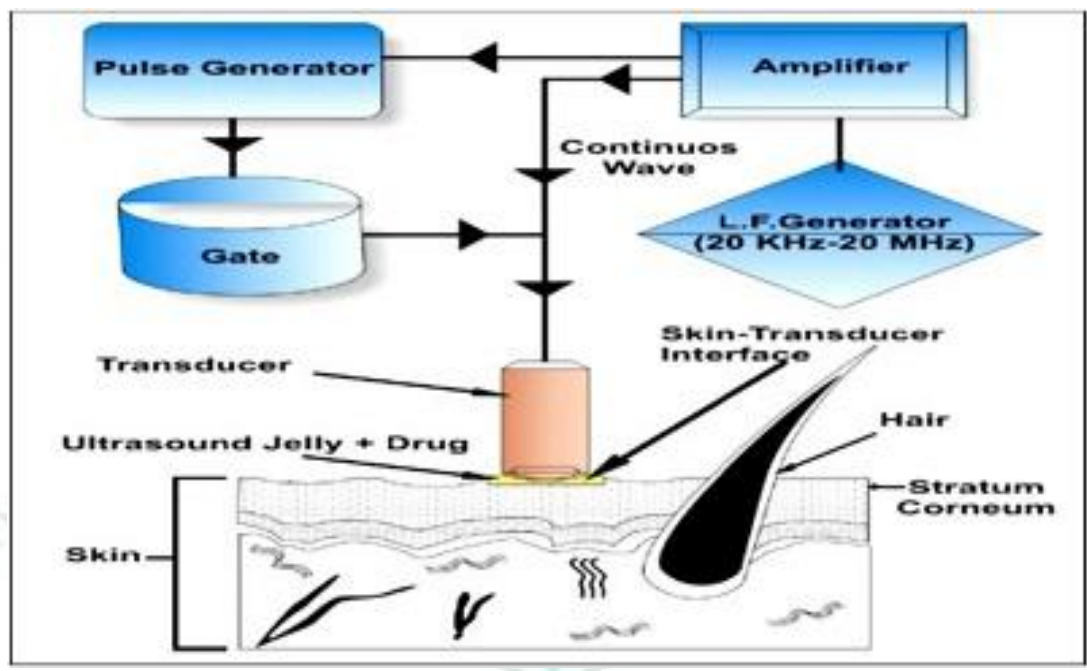

Figure 4: Diagramatic presentation of Sonophoresis ${ }^{12}$

\section{Eletroporation}

Electroporation is the phenomenon in which cell membrane permeability to ions and macromolecules is increased by exposing the cell to short high electric field pulses. The mechanism for electroporation by two pathways, through pores formed in the multiple lipid bilayer connecting corneocytes and through appendage cells. The efficacy of transport of drug depends on the electrical parameters and the physicochemical properties of drugs. In electroporation the transdermal delivery of the drug molecule is increased by the application of a high voltage (100 volts) in the form of direct currect (DC) $13,28,29$.

\section{Magnetophoresis}

This method involves the application of a magnetic field which acts as an external driving force to enhance the diffusion of a diamagnetic solute across the skin. Skin exposure to a magnetic field might also induce structural alterations that could contribute to an increase in permeability.

This technique can be used for the permeation of diamagnetic molecules. This method has been implemented for the transdermal delivery of benzoic acid. The diffusion flux is in direct proportion with intensity of the applied magnetic field.
Magnetoliposomes consist of magnetic Nanoparticles wrapped by a phospholipids bilayer which can be successfully applied for drug delivery systems, magnetic resonance imaging markers for cancer diagnosis, and thermal cancer therapy ${ }^{30,31,32}$.

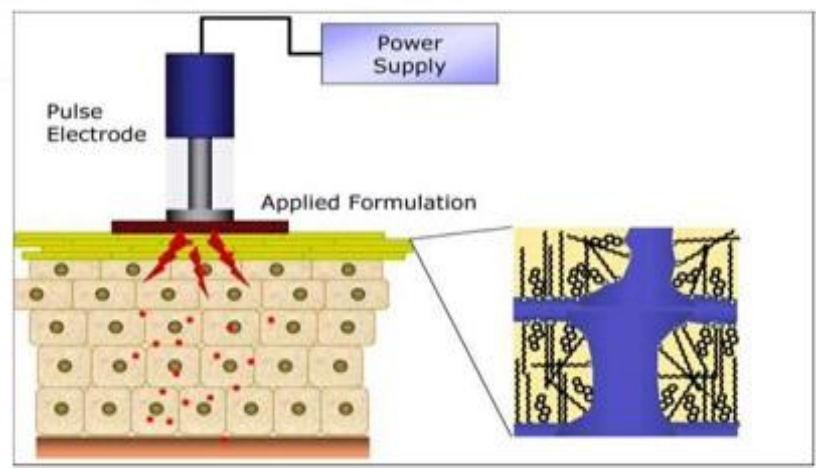

Figure 5: Diagrammatic presentation of Electroporation $^{31}$

\section{Microneedles}

Micrneedles technique may also be called as microscopic projection. As the name indiactes the device contains microprojections which are arranged in array.

The device as described in the patent consists of a drug reservoir and a plurality of projections extending from 
the reservoir. These microneedles of length 50 to 110 $\mathrm{mm}$ will penetrate the stratum corneum and epidermis to deliver the drug from the reservoir. The reservoir may contain drug, solution of drug, gel, or solid particulates, and the various embodiments of the invention include the use of a membrane to separate the drug from the skin and control release of the drug from its reservoir ${ }^{32-34}$.

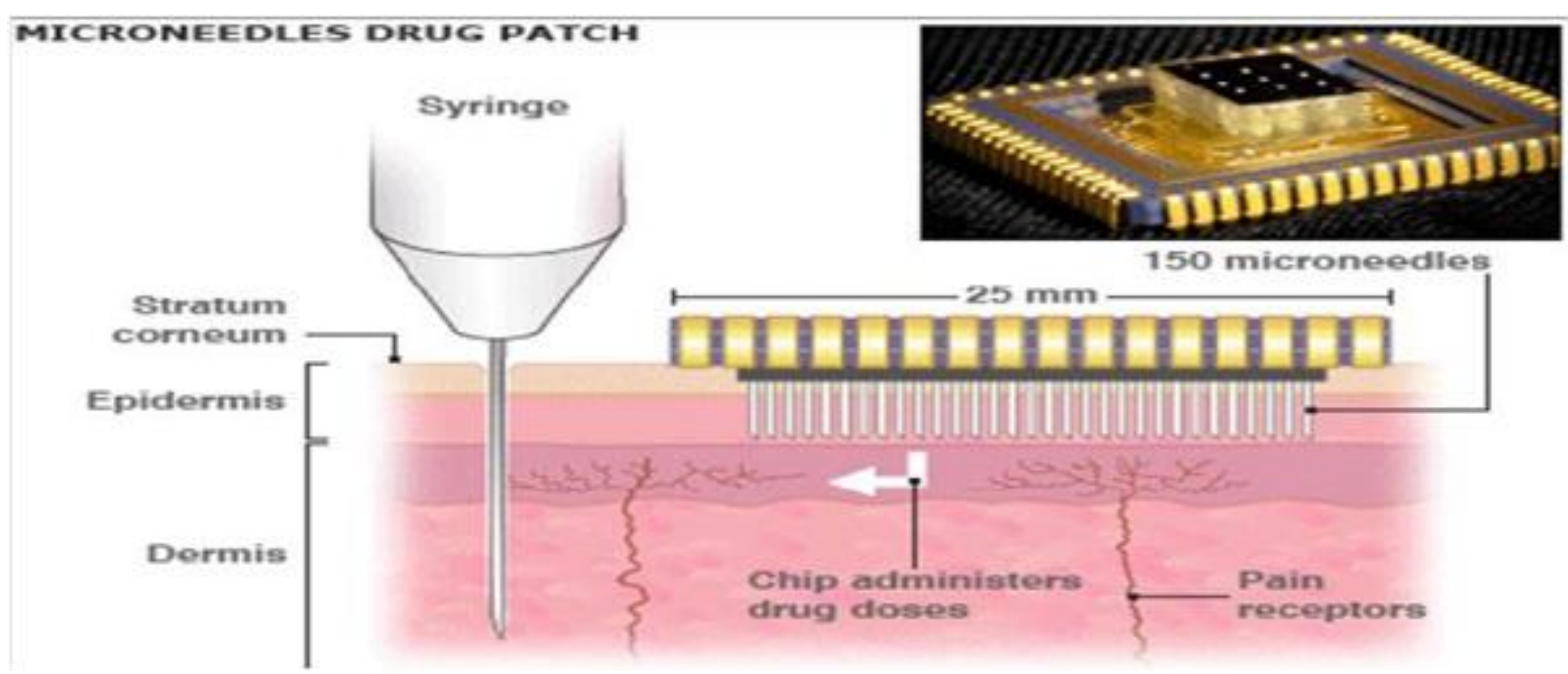

Figure 6: Microneedles based drug patch. ${ }^{14}$

\section{Radio - frequency}

Radio frequency involves the exposure of skin to high frequency alternating current $(\sim 100 \mathrm{kHz})$, resulting in the formation of heat-induced microchannels in the membrane in the same way as when laser radiation is employed. The rate of drug delivery is controlled by the number and depth of the microchannels formed by the device, which is dependent on the properties of the microelectrodes used in the device.

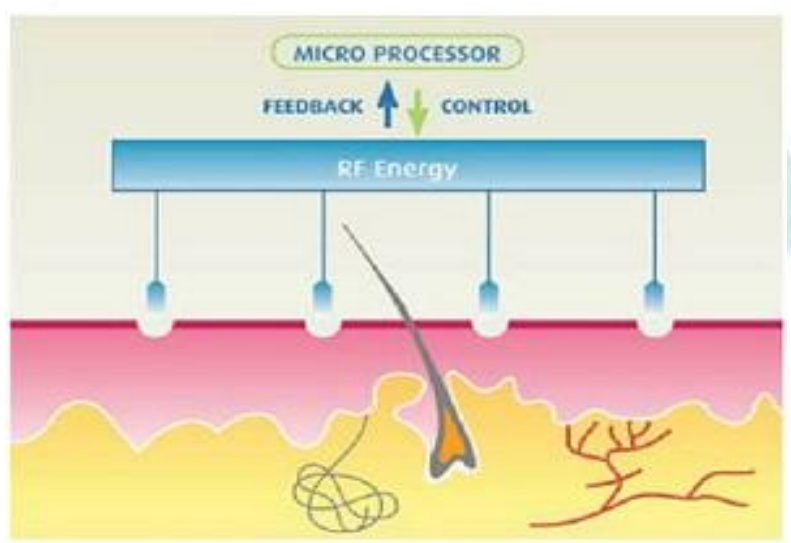

Figure 7: Diagrammatic presentation of radiofrequency for transdermal
The microneedle array is attached to the electronic device and placed in contact with the skin to facilitate the formation of the microchannels. Treatment duration takes less than a second, with a feedback mechanism incorporated within the electronic control providing a signal when the microchannels have been created, so as to ensure reproducibility of action. The drug patch is then placed on the treated area. It has been demonstrated from in vivo studies that plasma level of granisetron $\mathrm{HCl}$ after 12 hours was 30 times greater when exposed to radio frequency than the level recorded for untreated skin after 24 hours. In the same research permeability of diclofenac through the skin was also observed $^{14,36}$.

\section{CONCLUSION}

In order to overcome the skin's barrier function, the physical enhancements for the transdermal drugs have been developed. Physical techniques have made the penetration enhancer more effective. These approaches are very useful for the drugs having low permeable property, low soluble drugs and for the drugs having short biological half life. To overcome the problems of other techniques physical techniques has been found to be more effective as well as acceptable. 


\section{REFERENCES}

1. Jhawat VC, et al. "Transdermal drug delivery systems: approaches and advancements in drug absorption through skin." Int J Pharm Sci Rev Res. 2013; 20(1):47-56.

2. Bhowmik D, Dasari V, Duraivel S, Sampath Kumar KP. Recent Trends in Penetration Enhancer Used In Transdermal Drug Delivery System. The Pharma Innovation - Journal. Feb 2013; 2: 127-134.

3. Sharma A, Saini S, Rana AC, Transdermal Drug Delivery System: A Review. International Journal of research in pharmaceutical and biomedical sciences. 2013; 4(1):286-292.

4. Mezei M. Liposomes as penetration promoters and localizers of topically applied drugs. In Drug Permeation Enhancement: Theory and Applications, Hsieh, D.S., Ed.; Marcel Dekker: New York, NY, USA, 1994; pp. 171-198.

5. Ghafourian, T.; Samaras, E.G.; Brooks, J.D.; Riviere, J.E. Validated models for predicting skin penetration from different vehicles. Eur. J. Pharm. Sci. 2010; 41:612-616.

6. Kanikkannan $N$, Kandimalla $K$, Lamba SS, Singh $M$ Structures activity relationship of chemical penetration enhancers in transdermal drug delivery. Current Medicinal Chemistry 1999; 6:593-608.

7. Mbah, C. J., Uzor PF, Omeje O. "Journal of Chemical and Pharmaceutical Research." J. Chem. 2011; 3(3):680-700

8. Jaiswal PK, Kesharwani S, Kesharwani R, Patel DK, Ethosome: A new technology used as topical \& transdermal delivery system, Journal of Drug Delivery and Therapeutics. 2016; 6(3):7-17

9. Benson, Heather AE. "Transfersomes for transdermal drug delivery." Expert opinion on drug delivery. 2006; 3(6):727737.

10. Benson, Heather AE. "Transdermal drug delivery: penetration enhancement techniques." Current drug delivery 2.1 (2005): 23-33.

11. Morrow D.I.J. et al. "Innovative strategies for enhancing topical and transdermal drug delivery." The Open Drug Delivery Journal. 2007; 1:36-59.

12. Bavaskar, $\mathrm{K}$ et al. "The impact of penetration enhancers on transdermal drug delivery system: physical and chemical approach." Int J Phar Res Review. 2015; 4(7):14-24.

13. Saini S, Baghel S, Chauhan SS. "Recent development in penetration enhancers and techniques in transdermal drug delivery system." Journal of Advanced Pharmacy Education \& Research Jan-Mar 2014; 4.1.

14. Hussain, Abid, et al. "Potential Enhancers for Transdermal Drug Delivery: A Review." International Journal of Basic Medical Sciences and Pharmacy (IJBMSP). 2014; 4.1.

15. Prakash U.R.T., Thiagarajan P., Transdermal Drug Delivery Systems Influencing Factors, Study Methods and Therapeutic Applications, International Journal of Pharmacy, 2012; 2(2):366-374.

16. Ansel's Pharmaceutical Dosages Forms and Drug Delivery Systems, 9th Edition, Walters Kluwer (India) Pvt. Ltd., 2011, 294-311.

17. Ramteke K.H., Dhole S.N., Patil S.V., Transdermal Drug Delivery System: A Review, Journal of Advanced Scientific Research, 2012; 3(1):22-35.

18. Kumar R., Philip A., Modified Transdermal Technologies Breaking the Barrier of Drug Permeation via the Skin, Tropical Journal of Pharmaceutical Research, 2007; 6(1):633644.
19. Guy RH, Kalia YN, Delgado-Charro MB, Merino V, López A and Marro D, "Iontophoresis: electro repulsion and electro osmosis", J. Control. Rel., 2000; 64:129- 132.

20. Dhakar R C, Maurya SD, Saluja V, From formulation variables to drug entrapment efficiency of microspheres: a technical review, Journal of Drug Delivery and Therapeutics; 2012; 2(6):128-133.

21. Sachan R, Bajpai M. Transdermal drug delivery system: a review. Int. J. Res. \& Dev. Pharm. and Life Sci. Dec- Jan, 2013; 3(1):748-765.

22. Polat BE, Hart D, Langer R, Blankschtein, "Ultrasoundmediated transdermal drug delivery: mechanisms, scope, and emerging trends" J. Control. Release, 2011; 152:330-348.

23. Mormito Y, Mutoh M, Ueda H, Fang L, Hirayama K, Atobe M, Kobayashi D, "Elucidation of the transport pathway in hairless rat skin enhanced by low frequency sonophoresis based on the solute water transport relationship and confocal microscopy,” J. Control Release., 2005; 103:587-597.

24. Mitragotri S, Prausnitz MR, Langer R, "Current status and future potential of transdermal drug delivery," Nat Rev: Drug Discov., 2004; 3:115-124.

25. Samant LR, Bhaskar A. "Transdermal drug delivery system: Review." Journal of Pharmacy Research Vol. 2012; 5(2):899900 .

26. Shingade GM et al. "Review on: recent trend on transdermal drug delivery system. Journal of Drug Delivery and Therapeutics. 2012; 2(1):66-75.

27. Prausnitz MR, "A practical assessment of transdermal drug delivery by skin electroporation” Adv. Drug Deliv Rev., 1999; 35:61-76.

28. Tipre ND, Vavia RP. Formulation Optimization and Stability Study of Transdermal Therapeutic System of Nicorandil. Informa Healthcare 2002; 7(3):325-332.

29. Sugar IP, Neumann E. Stochastic model for electric fieldinduced membrane pores. Electroporation. Biophys. Chem.1984; 19(3):211-25.

30. Murthy SN, "Magnetophoresis: an approach to enhance transdermal drug diffusion," Pharmazie., 1999; 54:377-9

31. Jose Juan Escobar-Chavez, Isabel Marlen Rodriguez-Cruz, Clara Luisa Dominguez-Delgado. Chemical and Physical Enhancers for Transdermal Drug Delivery. Pharmacology. 2012; 978-953.

32. Tripathy S, Patel DK, Barob L, Naira SK, A review on phytosomes, their characterization, advancement \& potential for transdermal application, Journal of Drug Delivery and Therapeutics. 2013; 3(3):147-152

33. Gerstel MS, Place VA. Drug delivery device. US Pat 1976:964482. 89. Trautman J, Cormier MJ, Kim HL, Zuck MG. Device for enhancing a transdermal agent flux. US Pat 2000; 83:196

34. Trautman J, Wong PS, Daddona PE, Kim HL, Zuck MG. Device for enhancing transdermal agent flux. US Pat 2001:322808.

35. Yuzhakov VV, Sherman FF, Owens GD, Gartstein V. Apparatus and method for using an intracutaneous microneedle array. US Pat 2001:256533.

36. Sintov A, Krymbeck I, Daniel D, Hannan T, Sohn Z, Levin G, "Radiofrequency microchanneling as a new way for electrically assisted transdermal delivery of hydrophilic drugs", J. Control. Rel., 2003; 89:311-320. 